\title{
EXISTENCE DE SOLUTIONS SYMETRIQUES \\ POUR UN MODELE DE CHAMPS DE MESONS : LE MODELE D' ADKINS ET NAPPI
}

\author{
J. Dolbeault \\ CEREMADE \\ Université de Paris IX - Dauphine \\ Place du Maréchal de Lattre de Tassigny \\ 75775 Paris cedex 16 \\ et \\ Ecole Normale Supérieure \\ 45 , rue d'Ulm \\ 75230 Paris Cedex 05 \\ Communications in Partial Differential Equations \\ 15 (1990), no. 12,1743-1786
}

Résumé Dans cet article, nous étudions le modèle proposé par Adkins et Nappi pour décrire des configurations statiques de champs de mésons. Sous une hypothèse de symétrie, nous donnons une formulation précise d'un problème de minimisation avec contrainte de type degré topologique, et nous montrons l'existence de fonctions minimisantes. Nous présentons aussi le problème de minimisation sans hypothèse de symétrie.

Mots-clefs Problème de minimisation, degré, condition de symétrie.

\section{Plan}

I.Introduction

II.Formulation du modèle symétrique et choix d'un espace fonctionnel.

III.Minimisation pour le modèle symétrique.

IV.Le modèle non symétrique.

Appendice : degré et champs de mésons.

Bibliographie.

\section{Introduction}

Pour étudier l'interaction forte dans la limite de basse énergie, plusieurs approches sont possibles ; l'une d'elles consiste à construire des modèles de champs de mésons, et en particulier de pions (les pions sont les plus légères des particules soumises à l'interaction forte). Dans ces modèles, les baryons (en premier lieu, les nucléons : protons et neutrons) sont représentés à l'aide de solutions, dites solutions soliton, du champ des pions ; ces solutions sont obtenues en minimisant l'énergie associée au champ des pions, avec une contrainte de type degré topologique (physiquement, cette contrainte n'est autre que le nombre de baryons). Pour mener à bien les calculs numériques, on introduit en physique une hypothèse de symétrie, traditionnellement appelée ansatz de Skyrme. 
Un premier modèle a été présenté par Skyrme (voir $[\mathrm{S}],[\mathrm{A}, \mathrm{N}, \mathrm{W}]$ : dans ce modèle, l'énergie comporte un terme d'interaction non linéaire que l'on interprète comme un terme d'interaction du champ des pions avec un champ supplémentaire (ou plus exactement, comme la limite d'un terme d'interaction lorsque l'on fait tendre vers l'infini la masse des particules associées au champ supplémentaire). Le modèle de Skyrme a été étudié du point de vue mathématique par Esteban dans [E1-4] et par Esteban et Lions dans [E,L]. Ultérieurement au modèle de Skyrme, de nombreux autres modèles (voir $[\mathrm{A}, \mathrm{N}],[\mathrm{M}, \mathrm{K}, \mathrm{W}, \mathrm{W}],[\mathrm{M}, \mathrm{Z}],[\mathrm{V}, \mathrm{M}]$ ) pour lesquels le couplage avec des champs supplémentaires est explicite, ont été introduits à la suite d'Adkins et Nappi. Cet article a pour objet l'étude, du point de vue mathématique, du modèle proposé par Adkins et Nappi dans $[A, N]$ : on montrera l'existence d'une solution lorsque l'on se restreint au modèle avec hypothèse de symétrie (ansatz de Skyrme) et on indiquera quelques unes des difficultés qui apparaissent si on ne fait pas cette hypothèse.

Les champs du modèle d'Adkins et Nappi sont des fonctions $\phi=\left(\phi^{0}, \phi^{1}, \phi^{2}, \phi^{3}\right)$ définies sur $\mathbb{R}^{3}$ et à valeurs dans la sphère unité $S^{3}$ de $\mathbb{R}^{4}$, couplées à un champ scalaire $\Omega$ défini sur $\mathbb{R}^{3}$, pour lesquelles l'énergie est donnée par :

$$
E[\phi]=\|\nabla \phi\|_{L^{2}}^{2}+\|\Omega\|_{H^{1}}^{2}
$$

et où le couplage est défini par l'équation :

$$
-\Delta \Omega+\Omega=\operatorname{det}(\phi, \nabla \phi) .
$$

En négligeant pour l'instant le problème délicat et important du choix d' un espace fonctionnel approprié pour $\phi$ (et pour $\Omega$ ), on voit que $\Omega$ est défini de manière unique par l'équation (2), en supposant par exemple qu'à l'infini $\Omega$ est assez petit dans un sens faible : $E$ est donc une fonctionnelle portant sur $\phi$ uniquement.

On définit aussi :

$$
d[\phi]=\frac{1}{2 \pi^{2}} \int_{\mathbb{R}^{3}} \operatorname{det}(\phi, \nabla \phi) d x .
$$

Nous verrons dans l'appendice que lorsque $\phi$ est de classe $C^{1}$, constante en dehors d'un compact, $d[\phi]$ coïncide avec le degré usuel (proposition A2), et que, plus généralement, si $\nabla \phi$ est $L^{2}$, l'inégalité de Poincaré-Wirtinger montre que $\phi$ est constante au voisinage de l'infini dans un sens faible (proposition A1) : la définition (3) fournit alors - sous réserve qu'elle ait un sens - un moyen d'étendre la notion de degré.

Le problème qui se pose alors est de savoir si l'infimum suivant est atteint :

$$
I_{k}=\inf \{E[\phi] \mid \phi \in X \text { et } d[\phi]=k\} \quad(k \in)
$$

où $X$ désigne un espace fonctionnel convenable sur le choix duquel nous reviendrons plus loin, et on dira qu'une fonction $\phi$ de $X$ est solution du modèle d'Adkins et Nappi si :

$$
d[\phi]=k \quad \text { et } \quad E[\phi]=I_{k} \quad .
$$

En fait, nous allons reformuler le modèle d'Adkins et Nappi dans le cadre de l'ansatz de Skyrme et nous ne répondrons tout d'abord aux questions soulevées par le modèle que dans ce cadre.

L'ansatz de Skyrme consiste à ne prendre en compte que des fonctions de la forme :

$$
\forall x \in \mathbb{R}^{3} \quad \phi(x)=\left(\cos \theta(r), \frac{x}{r} \sin \theta(r)\right)
$$


où on a utilisé la notation :

$$
r=|x|
$$

On notera une telle fonction $\phi_{\theta}$, et nous parlerons de modèle symétrique dès lors que nous nous restreindrons à des fonctions de ce type.

La fonction $\phi_{\theta}$ sera solution du modèle d'Adkins et Nappi symétrique lorsqu'elle réalisera, sur l'ensemble des fonctions vérifiant l'ansatz de Skyrme, l'infimum de $E[\phi]$ pour $d[\phi]=k$.

\section{Notations}

Suivant le contexte, $|\cdot|$ désigne la valeur absolue, la norme euclidienne usuelle, ou la mesure de Lebesgue. On notera $B(x, R)$ et $B(R)=B(0, R)$ les boules ouvertes de $\mathbb{R}^{3}$. En général, on désignera les espaces fonctionnels usuels simplement par : $H^{1}\left(\mathbb{R}^{3}\right), L^{p}\left(\mathbb{R}^{3}\right), L^{q}(] 0, \infty[), \ldots$ et on notera : $\|\cdot\|_{H^{1}},\|\cdot\|_{L^{p}},\|\cdot\|_{L^{q}}, \ldots$ leurs normes respectives. On notera $r=|x|$ pour tout point $x$ de $\mathbb{R}^{3}$.

$\left(e_{i}\right)_{i=1,2,3,4}$ est la base canonique de $\mathbb{R}^{4}$.

Les dérivées seront souvent désignées, de manière simplifiée par :

$$
\begin{gathered}
\partial_{\alpha} \phi=\frac{\partial \phi}{\partial x^{\alpha}} \quad(\alpha=1,2,3), \\
\dot{\theta}(r)=\frac{d \theta}{d r} .
\end{gathered}
$$

Enfin, on notera $\delta$ la distribution de Dirac en $x=0 \in \mathbb{R}^{3}$.

\section{Formulation du modèle symétrique et choix d'un espace fonctionnel}

Nous allons étudier l'ansatz de Skyrme, puis énoncer quelques propriétés des fonctions vérifiant cet ansatz et formuler le problème de minimisation associé au modèle symétrique de manière précise.

Considérons une fonction $\theta$ définie sur $[0, \infty$ [, à valeurs réelles, que nous supposerons pour l'instant de classe $C^{1}$ sur $[0, \infty[$, à support compact, telle que :

$$
\theta(0) \in \pi
$$

et définissons la fonction $\phi_{\theta}$ sur $\mathbb{R}^{3}$ par :

$$
\forall x \in \mathbb{R}^{3} \quad \phi_{\theta}(x)=\left(\cos \theta(r), \frac{x}{r} \sin \theta(r)\right)
$$


Définissons maintenant les fonctionnelles $E^{*}$ et $d^{*}$ par :

$$
\begin{aligned}
E^{*}[\theta] & =\frac{1}{4 \pi} E\left[\phi_{\theta}\right] \\
d^{*}[\theta] & =d\left[\phi_{\theta}\right] .
\end{aligned}
$$

On montre aisément que :

$$
\forall x \in \mathbb{R}^{3} \quad \operatorname{det}\left(\phi_{\theta}, \nabla \phi_{\theta}\right)(x)=-\frac{1}{r^{2}} \sin ^{2} \theta(r) \cdot \dot{\theta}(r)
$$

en désignant la dérivée de $\theta$ par $\dot{\theta}$. On obtient en particulier :

$$
\begin{gathered}
d^{*}[\theta]=-\frac{2}{\pi} \int_{0}^{\infty} \sin ^{2} \theta \cdot \dot{\theta} d r \\
d^{*}[\theta]=-\frac{2}{\pi}[P(\theta(r))]_{0}^{\infty}
\end{gathered}
$$

où $P$ est la fonction réelle (continue, inversible, d'inverse continue) définie par :

$$
\forall \theta \in \mathbb{R} \quad P(\theta)=\frac{1}{2} \theta-\frac{1}{4} \sin (2 \theta) .
$$

Par ailleurs l'équation (2) peut être réécrite pour $\phi=\phi_{\theta}$ sous la forme

$$
-\Delta \Omega+\Omega=-\frac{1}{r^{2}} \sin ^{2} \theta \cdot \dot{\theta}
$$

et il est naturel de chercher des solutions sous la forme :

$$
\Omega(x)=\omega(r) \quad\left(\forall x \in \mathbb{R}^{3}\right), \quad \text { avec } r=|x|
$$

L'équation (2) devient alors, sur ]0, $\infty[$ :

$$
-\frac{d}{d r}\left(r^{2} \frac{d \omega}{d r}\right)+r^{2} \omega+\sin ^{2} \theta \cdot \frac{d \theta}{d r}=0 .
$$

En remarquant que :

$$
\left|\nabla \phi_{\theta}\right|^{2}(x)=\dot{\theta}^{2}+\frac{2}{r^{2}} \sin ^{2} \theta \quad\left(\forall x \in \mathbb{R}^{3}-\{0\}\right),
$$

on obtient l'expression :

$$
E^{*}[\theta]=\int_{0}^{\infty}\left\{r^{2} \dot{\theta}^{2}+2 \sin ^{2} \theta+r^{2}\left(\omega^{2}+\dot{\omega}^{2}\right)\right\} d r
$$

et comme précédemment, on peut poser le problème de minimisation :

$$
I_{k}^{*}=\inf \left\{E^{*}[\theta] \mid \theta \in X^{*} \text { et } d^{*}[\theta]=k\right\} \quad(k \in)
$$

où $X^{*}$ est un espace fonctionnel convenable, défini plus loin.

Lemme 1 Soit $\varphi \in H_{l o c}^{1}(] 0, \infty[)$ tel que : $r \dot{\varphi} \in L^{2}(] 0, \infty[)$. Notons :

$I=\left(\int_{0}^{\infty} r^{2} \dot{\varphi}^{2} d r\right)^{1 / 2}$. Alors il existe une fonction $\tilde{\varphi}$, continue sur $] 0, \infty[$, possédant une limite : $\varphi(\infty)=\lim _{r \rightarrow+\infty} \tilde{\varphi}(r)$, qui vérifie les propriétés suivantes : 
(i) Pour presque tout $r>0 \quad \varphi(r)=\tilde{\varphi}(r)$

(ii) $|\tilde{\varphi}(t)-\tilde{\varphi}(s)| \leq I \cdot\left(\frac{|t-s|}{t s}\right)^{1 / 2} \quad(\forall(t, s) \in] 0, \infty\left[^{2}\right)$

Preuve Considérons la fonction $\hat{\varphi}$ définie par :

$$
\forall r \in] 0, \infty\left[\quad \hat{\varphi}(r)=\int_{1}^{r} \dot{\varphi} d s\right.
$$

Il existe un réel $\alpha$ tel que $\hat{\varphi}(r)=\varphi(r)+\alpha$ presque partout.

$\dot{\varphi} \in L_{l o c}^{1}(] 0, \infty[): \hat{\varphi}$ est continue sur $] 0, \infty[$.

Soit $0<t \leq s$ :

$$
\begin{aligned}
|\hat{\varphi}(s)-\hat{\varphi}(t)| & =\left|\int_{t}^{s} \hat{\varphi}(r) d r\right| \\
& \leq\left|\int_{t}^{s} r^{2} \dot{\varphi}^{2} d r \cdot \int_{t}^{s} \frac{1}{r^{2}}\right|^{1 / 2} \\
& \quad \text { par l'inégalité de Cauchy }- \text { Schwarz } \\
& \leq \sqrt{\frac{s-t}{t s}} \cdot I
\end{aligned}
$$

De plus :

$$
\frac{s-t}{t s} \leq \frac{s}{t s}=\frac{1}{t}
$$

ce qui assure, par le critère de Cauchy, l'existence de :

$$
\lim _{r \rightarrow \infty}(\hat{\varphi}(r)-\alpha)=\varphi(\infty) \quad .
$$

Le lemme est alors démontré, en définissant la fonction $\tilde{\varphi}$ par :

$$
\left.\tilde{\varphi}(r)=\varphi(\infty)-\int_{r}^{\infty} \dot{\varphi}(s) d s \quad \forall r \in\right] 0, \infty[\quad .
$$

Remarque 1 Avec les notations utilisées dans l'introduction, le lemme 1 s'applique aussi bien au cas $\varphi=\theta$ qu'au cas : $\varphi=\omega$. Dans le premier cas, on suppose que : $\int_{0}^{\infty} \sin ^{2} \theta(r) d r$ converge, ce qui assure que :

$$
\theta(\infty) \in \pi \cdot .
$$

Dans le second cas, on suppose que : $\int_{0}^{\infty} r^{2} \omega^{2}(r) d r$ converge, ce qui assure que :

$$
\omega(\infty)=0
$$

Dans la suite, aussi bien pour $\varphi=\theta$ que pour $\varphi=\omega$, nous confondrons $\varphi$ et $\tilde{\varphi}$, ce qui revient à choisir le représentant continu dans la classe de fonctions considérée. Par contre, les équations différentielles continueront d'être écrites au sens presque partout.

Proposition 1 Soit $\theta \in H_{l o c}^{1}(] 0, \infty[) \cap C^{0}(] 0, \infty[)$ tel que :

$$
r \dot{\theta} \in L^{2}(] 0, \infty[) \quad \text { et } \quad \sin \theta \in L^{2}(] 0, \infty[) \quad .
$$

Supposons que $\theta$ est continue en $r=0+$. Alors $d^{*}[\theta]$ vaut : 


$$
d^{*}[\theta]=\frac{2}{\pi} \cdot P(\theta(0))-\frac{1}{\pi} \theta(\infty)
$$

Preuve Compte-tenu du lemme 1 et de la remarque 1, la limite :

$$
\lim _{r \rightarrow \infty} \theta(r)=\theta(\infty)
$$

existe et :

$$
\theta(\infty) \in \pi
$$

donc :

$$
\begin{aligned}
P(\theta(\infty)) & =\frac{1}{2} \theta(\infty)-\frac{1}{4} \sin (2 \theta(\infty)) \\
& =\frac{1}{2} \theta(\infty)
\end{aligned}
$$

ce qui démontre immédiatement la proposition 1.

Remarque 2 Soit $\theta$ une fonction vérifiant les hypothèses de la proposition 1. Si l'on suppose que la fonction $\phi_{\theta}$ définie par (4) est continue en $x=0 \in \mathbb{R}^{3}$, alors $\theta(0)$ est multiple de $\pi$ et $d^{*}[\theta]$ est entier : $d^{*}[\theta]$ est en fait le degré, au sens usuel, de $\phi_{\theta}$ (voir la proposition A3 de l'appendice). Dans la suite, on choisira un cadre fonctionnel plus large, et on supposera simplement, comme dans les hypothèses de la proposition 1 , que $\theta$ est continue en $r=0+$.

Proposition 2 Soit $\theta \in H_{l o c}^{1}(] 0, \infty[) \cap C^{0}(] 0, \infty[)$ tel que :

$$
r \dot{\theta} \in L^{2}(] 0, \infty[)
$$

Alors :

(i) Toute solution de :

$$
-\frac{d}{d r}\left(r^{2} \dot{\omega}\right)+r \omega+\sin ^{2} \theta \dot{\theta}=0
$$

s'écrit sous la forme :

$$
\omega(r)=\omega_{0}(r)+A \frac{e^{-r}}{r}+B \frac{e^{r}}{r} \quad(r \in] 0, \infty[p . p .)
$$

où $A$ et $B$ sont des constantes réelles et où :

$$
\begin{aligned}
\omega_{0}(r)=- & \frac{e^{r}}{r}\left[P(\theta(r))+\int_{0}^{r} \sin ^{2} \theta(s) \dot{\theta}(s)\left(\frac{s h s}{s}-1\right) d x\right] \\
& -\frac{s h r}{r} \int_{r}^{\infty} \sin ^{2} \theta(s) \dot{\theta}(s) \frac{e^{-s}}{s} d s
\end{aligned}
$$

(ii) $\omega_{0}$ est de classe $C^{2}$ et :

$$
\begin{aligned}
\dot{\omega}_{0}(r)= & \frac{e^{-r}}{r}\left(1+\frac{1}{r}\right)\left[P(\theta(r))+\int_{0}^{r} \sin ^{2} \theta(s) \dot{\theta}(s)\left(\frac{s h s}{s}-1\right) d s\right] \\
& -\frac{r \cdot \operatorname{ch} r-s h r}{r^{2}} \int_{r}^{\infty} \sin ^{2} \theta(s) \dot{\theta}(s) \frac{e^{-s}}{s} d s .
\end{aligned}
$$

(iii) les assertions suivantes sont équivalentes :

(a) $\theta$ est continue en: $r=0+$ et $\frac{1}{r}[P(\theta(r))-P(\theta(0))] \in L^{2}(] 0, \infty[)$. 
(b) Il existe une solution $\omega$ de (10) telle que :

$$
r \omega \in L^{2}(] 0, \infty[) \quad \text { et } \quad r \dot{\omega} \in L^{2}(] 0, \infty[)
$$

(iv) Si l'une des deux assertions (a) ou (b) de (iii) est vérifiée, alors il existe une solution unique $\omega$ de (10), qui s'écrit :

$$
\omega(r)=\omega_{0}(r)+A \frac{e^{-r}}{r} \quad(r \in] 0, \infty[p . p .) \quad \text { avec } A=P(\theta(0))
$$

La remarque 2, sur le choix du représentant de la classe de fonctions, s'applique encore, et aussi bien pour (15) que pour (18), nous supposerons que $\omega$ est continue.

Preuve Soit $\theta$ une fonction vérifiant les hypothèses de la proprosition 2. Remarquons pour commencer que $\omega_{0}$ est bien définie pour tout réel $r>0$. En effet, pour tout réel $s>0$, on a :

$$
s .\left|\sin ^{2} \theta(s) \dot{\theta}(s)\right| \leq|s \cdot \dot{\theta}(s)| \text {. }
$$

En définissant $g$ par : $g(s)=\frac{1}{s^{2}}\left(\frac{s h s}{s}-1\right)$ et en utilisant l'in'egalité de Cauchy-Schwarz, on obtient

$$
\left[\int_{0}^{r} \sin ^{2} \theta(s) \dot{\theta}(s)\left(\frac{s h s}{s}-1\right) d s\right]^{2} \leq \int_{0}^{r} s^{2} \dot{\theta}^{2}(s) d s \cdot \int_{0}^{r} s^{2} g^{2}(s) d s
$$

Par l'inégalité de Cauchy-Schwarz, on obtient également :

$$
\left[\int_{r}^{\infty} \sin ^{2} \theta(s) \dot{\theta}(s) \frac{e^{-s}}{s} d s\right]^{2} \leq \int_{r}^{\infty} s^{2} \dot{\theta}^{2}(s) d s \cdot \int_{r}^{\infty}\left(\frac{e^{-s}}{s^{2}}\right)^{2} d s
$$

Les inégalités (19a) et (19b) assurent que $\omega_{0}$ est définie sur $] 0, \infty[$.

On vérifie aisément qu'au sens des distributions, $\dot{\omega}_{0}$ s'écrit sous la forme (17), et donc que $\omega_{0}$ est de classe $C^{1}$; en fait, comme $\omega_{0}$ est solution de $(10), \omega_{0}$ est de classe $C^{2}$ sur $] 0, \infty[$, ce qui démontre (ii).

L'équation différentielle homogène associée à (10) s'écrit :

$$
-\frac{d}{d r}\left(r^{2} \dot{\omega}\right)+r^{2} \omega=0
$$

et l'on vérifie aisément que ses solutions sont de la forme :

$$
\omega(r)=A \frac{e^{-r}}{r}+B \frac{e^{r}}{r},
$$

où $A$ et $B$ sont deux constantes réelles, ce qui démontre (i).

Nous allons maintenant considérer les équivalences de $\omega_{0}$ au voisinage de $r=0+$ et au voisinage de $r=+\infty$.

$\underline{\text { Au voisinage de } r=0+\text { : }}$

$$
\int_{0}^{r} s^{2} g^{2}(s) d s=0\left(r^{5 / 2}\right)
$$


ce qui assure par (19a) que:

$$
\begin{gathered}
\int_{0}^{r} \sin ^{2} \theta(s) \dot{\theta}(s)\left(\frac{s h s}{s}-1\right) d s=0\left(r^{5 / 4}\right) . \\
\int_{r}^{\infty} \sin ^{2} \theta(s) \dot{\theta}(s) \frac{e^{-s}}{s} d s=-P(\theta(r)) \frac{e^{-r}}{r}+\int_{r}^{\infty} P(\theta(s)) \frac{e^{-s}}{s}\left(1+\frac{1}{s}\right) d s,
\end{gathered}
$$

en intégrant par parties. Si (a) est vérifiée :

$$
\int_{r}^{\infty} P(\theta(s)) \frac{e^{-s}}{s}\left(1+\frac{1}{s}\right) d s=0\left(\frac{P(\theta(0))}{r}\right),
$$

ce qui assure que :

$$
\int_{r}^{\infty} \sin ^{2} \theta(s) \dot{\theta}(s) \frac{e^{-s}}{s} d s=0\left(\frac{P(\theta(0))}{r}\right) .
$$

Finalement, si (a) est vérifiée, on a :

$$
\begin{gathered}
\omega_{0}(r)+\frac{e^{-r}}{r} P(\theta(r))=0\left(r^{1 / 4}\right)+0\left(\frac{P(\theta(0))}{r}\right), \\
\dot{\omega}_{0}(r)-\frac{e^{-r}}{r}\left(1+\frac{1}{r}\right) P(\theta(r))=0\left(r^{-3 / 4}\right)+0(P(\theta(0)) .
\end{gathered}
$$

Au voisinage de $r=+\infty$ :

$$
\begin{gathered}
\int_{0}^{r} \sin ^{2} \theta(s) \dot{\theta}(s)\left(\frac{s h s}{s}-s\right) \\
=P(\theta(s))\left(\frac{s h r}{r}-1\right)-\int_{0}^{r} P(\theta(s)) \frac{s \cdot c h s-s h s}{s^{2}} d s \\
\frac{1}{s^{2}}(s \cdot \text { chs }-s h s) \leq \frac{e^{s}}{r} \quad \text { pour } s \geq r, \text { donc }: \\
\int_{0}^{r} \sin ^{2} \theta(s) \dot{\theta}(s)\left(\frac{s h s}{s}-1\right) d s=0\left(\frac{e^{r}}{r}\right) \\
\int_{r}^{\infty} P(\theta(s)) \frac{e^{-s}}{s}\left(1+\frac{1}{s}\right) d s=0\left(\frac{e^{-r}}{r}\right), \operatorname{donc}: \\
\int_{r}^{\infty} \sin ^{2} \theta(s) \dot{\theta}(s) \frac{e^{-s}}{s} d s=0\left(\frac{e^{-r}}{r}\right)
\end{gathered}
$$

Finalement :

$$
\begin{gathered}
\omega_{0}(r)=0\left(\frac{1}{r^{2}}\right), \\
\dot{\omega}_{0}=0\left(\frac{1}{r^{2}}\right) .
\end{gathered}
$$

Soit $\omega$ une solution de (10) :

$$
\omega(r)=\omega_{0}(r)+A \frac{e^{-r}}{r}+B \frac{e^{r}}{r}
$$

Au voisinage de $r=+\infty, \omega(r) \sim B \frac{e^{r}}{r}$ si $B \neq 0$, donc $r \omega \in L^{2}(] 0, \infty[)$ si et seulement si :

$$
B=0 .
$$

Supposons maintenant que (a) est vérifiée. En écrivant : 


$$
\frac{e^{-r}}{r}(-P(\theta(r))+A)=\frac{e^{-r}}{r}(P(\theta(0))-P(\theta(r)))+\frac{e^{-r}}{r}(A-P(\theta(0)))
$$

on voit, en utilisant les équivalents obtenus au voisinage de $r=0+$,

que : $r \dot{\omega}(r) \in L^{2}(] 0, \infty[)$ si et seulement si :

$$
A=P(\theta(0)),
$$

et donc :

$$
\omega(r)=\omega_{0}(r)+P(\theta(0)) \frac{e^{-r}}{r},
$$

ce qui démontre (iv), et aussi l'implication : (a) $\Longrightarrow$ (b) de l'assertion (iii).

Il reste donc à montrer l'implication : (b) $\Longrightarrow(\mathrm{a})$.

Supposons qu'il existe une solution $\omega$ de (10), telle que :

$$
r \omega \in L^{2}(] 0, \infty[) \quad \text { et } \quad r \dot{\omega} \in L^{2}(] 0, \infty[) \quad .
$$

Par (15) et (20), $\omega$ s'écrit sous la forme :

$$
\omega(r)=\omega_{0}(r)+A \frac{e^{-r}}{r}
$$

Montrons que :

$$
\lim _{r \rightarrow 0+} P(\theta(r))=A .
$$

Soit deux réels $r_{1}$ et $r_{2}$ tels que : $0<r_{1}<r_{2}$

$$
\begin{aligned}
\left|\left[(P(\theta(r))-A)^{2}\right]_{r_{1}}^{r_{2}}\right|=\mid & \int_{r_{1}}^{r_{2}}(P(\theta(r))-A) \cdot P^{\prime}(\theta) \cdot \dot{\theta}(r) d r \mid \\
\leq & \left(\int_{r_{1}}^{r_{2}}\left(\frac{P(\theta(r))-A}{r}\right)^{2} d r \cdot \int_{r_{1}}^{r_{2}} r^{2} \dot{\theta}^{2}(r) d r\right)^{1 / 2} \\
& \text { par l'inégalité de Cauchy - Schwarz. }
\end{aligned}
$$

Au voisinage de $r=0+$, on a :

$$
\begin{aligned}
\left(\frac{P(\theta(r))-A}{r}\right)^{2} \sim r^{2} & {\left[(P(\theta(r))-A) \frac{e^{-r}}{r}\left(1+\frac{1}{r}\right)\right]^{2} } \\
= & {\left[r \dot{\omega}(r)-\left(r \dot{\omega}_{0}(r)-P(\theta(r)) \frac{e^{-r}}{r}\left(1+\frac{1}{r}\right)\right)\right]^{2} }
\end{aligned}
$$

or :

$$
r \dot{\omega} \in L^{2}(] 0, \infty[)
$$

et :

$$
\begin{aligned}
r \dot{\omega}_{0}(r) & -P(\theta(r)) \frac{e^{-r}}{r}\left(1+\frac{1}{r}\right) \\
& =\frac{r \cdot \operatorname{ch} r-s h r}{r} \cdot \int_{r}^{\infty} \sin ^{2} \theta(s) \dot{\theta}(s) \frac{e^{-r}}{r} d s .
\end{aligned}
$$

Comme :

$$
\frac{r \cdot \operatorname{ch} r-s h r}{r}=0\left(r^{2}\right)
$$


et :

$$
\begin{aligned}
\left|\int_{r}^{\infty} \sin ^{2} \theta(s) \dot{\theta}(s) \frac{e^{-s}}{s} d s\right| & \leq\left(\int_{0}^{\infty} s^{2} \dot{\theta}^{2} d s \cdot \int_{r}^{\infty} \frac{e^{-2 s}}{s^{4}} d s\right)^{1 / 2} \\
& =0\left(r^{-3 / 2}\right),
\end{aligned}
$$

on est assuré que :

$$
\frac{P(\theta(r))-A}{r} \in L^{2}(] 0, \infty[)
$$

Le critère de Cauchy assure l'existence de :

$$
\lim _{r \rightarrow 0+}[P(\theta(r))-A]
$$

et la convergence de :

$$
\int_{0}^{\infty}\left(\frac{P(\theta(r))-A}{r}\right)^{2} d r
$$

démontre (21). La fonction $P(\theta)$ est donc continue en $r=0+$, et $P(\theta)$ étant inversible, d'inverse continue, $\theta$ est continue en $r=0+$, ce qui achève la démonstration de (iii).

Les propositions 1 et 2 justifient l'introduction de l'espace fonctionnel suivant :

$$
\begin{aligned}
X^{*}= & \left\{\theta \in H_{l o c}^{1}(] 0, \infty[) \cap C^{0}\left(\left[0, \infty[) \mid r \dot{\theta} \in L^{2}(] 0, \infty[),\right.\right.\right. \\
& \left.\sin \theta \in L^{2}(] 0, \infty[), \frac{1}{r}[P(\theta(r))-P(\theta(0))] \in L^{2}(] 0, \infty[)\right\}
\end{aligned}
$$

En effet, les fonctionnelles $E^{*}$ et $d^{*}$ sont bien définies sur $X^{*}$, au sens suivant :

$$
\begin{gathered}
d^{*}[\theta]=\frac{2}{\pi}[P(\theta(r))]_{0}^{\infty} \\
E^{*}[\theta]=\int_{0}^{\infty}\left\{r^{2} \dot{\theta}^{2}+2 \sin ^{2} \theta+r^{2}\left(\omega^{2}+\dot{\omega}^{2}\right\} d r\right.
\end{gathered}
$$

le couplage entre $\theta$ et $\omega$ étant assuré par l'équation (10) :

$$
-\frac{d}{d r}\left(r^{2} \dot{\omega}\right)+r^{2} \omega+\sin ^{2} \theta \dot{\theta}=0
$$

que l'on résoud par :

$$
\omega=\omega_{0}+P(\theta(0)) \frac{e^{-r}}{r}
$$

avec :

$$
\begin{aligned}
\omega_{0}(r)=- & \frac{e^{-r}}{r}\left[P(\theta(r))+\int_{0}^{r} \sin ^{2} \theta(s) \dot{\theta}(s)\left(\frac{s h s}{s}-1\right) d s\right] \\
& -\frac{s h r}{r} \int_{r}^{\infty} \sin ^{2} \theta(s) \dot{\theta}(s) \frac{e^{-s}}{s} d s .
\end{aligned}
$$

Comme nous l'avons remarqué précédemment, la fonctionnelle $d^{*}$ n'est pas nécessairement à valeurs entières. En fait, $d^{*}$ peut prendre n'importe quelle valeur $\alpha$ réelle. Considérons en effet une fonction :

$$
\theta \in C^{0}\left(\left[0, \infty[) \cap C^{\infty}(] 0, \infty[),\right.\right.
$$

à support compact, telle que :

$$
\begin{array}{ll}
\theta(r)=\theta_{0} & \forall r \in[0,1] \\
\theta(r)=0 & \forall r \in[2, \infty]
\end{array}
$$

$\theta_{0}$ étant l'unique réel tel que $P\left(\theta_{0}\right)=\frac{\alpha \pi}{2}$. Il est aisé de montrer que : $\theta \in X^{*}$ et $d^{*}[\theta]=\alpha$. 
Nous sommes donc en mesure de considérer l'infimum suivant :

$$
I_{\alpha}^{*}=\inf \left\{E^{*}[\theta] \mid \theta \in X^{*} \text { et } d^{*}[\theta]=\alpha\right\}
$$

\section{Minimisation pour le modèle symétrique}

Nous allons montrer le principal résultat de cet article : l'infimum $I_{\alpha}^{*}$ est atteint pour tout réel $\alpha$.

Commençons par la remarque suivante : si $\theta \in X^{*}$, alors :

$$
(\theta+2 k \pi) \in X^{*} \quad \forall k \in \quad .
$$

De plus :

$$
\begin{aligned}
E^{*}[\theta+2 k \pi] & =E^{*}[\theta] \\
d^{*}[\theta+2 k \pi] & =d^{*}[\theta]
\end{aligned}
$$

ce qui permet de réécrire $I_{\alpha}^{*}$ sous la forme :

$$
I_{\alpha}^{*}=\inf \left\{E^{*}[\theta] \mid \theta \in X^{*}, \theta(\infty)=0, P(\theta(0))=\frac{\alpha \pi}{2}\right\} .
$$

La condition sur le degré $d^{*}$ se réduit en fait - dans le cadre de l'ansatz de Skyrme - à une condition sur les limites de $\theta$ en $0+$ et en $+\infty$.

En utilisant l'équation (10), on peut réécrire l'énergie sous la forme :

$$
\begin{gathered}
E^{*}[\theta]=e^{*}[\theta, \omega] \\
e^{*}[\theta, \omega]=\int_{0}^{\infty}\left\{r^{2} \dot{\theta}^{2}+2 \sin ^{2} \theta-2 \omega \sin ^{2} \theta \dot{\theta}-r^{2}\left(\omega^{2}+\dot{\omega}^{2}\right)\right\} d r
\end{gathered}
$$

et sous cette forme, il est clair que le minimum de l'énergie $E^{*}[\theta]$, lorsque $\theta$ et $\omega$ sont couplés par l'équation (10), est en fait un point critique de la fonctionnelle $e^{*}[\theta, \omega]$. Comme la condition sur le degré est une condition aux limites, il est possible d'écrire un système d'équations d'Euler-Lagrange :

$$
\begin{aligned}
& -\frac{d}{d r}\left(r^{2} \dot{\omega}\right)+r^{2} \omega+\sin ^{2} \theta \dot{\theta}=0 \\
& -\frac{d}{d r}\left(r^{2} \dot{\theta}\right)+\sin (2 \theta)+\sin ^{2} \theta \dot{\omega}=0
\end{aligned}
$$

pour toute fonction $\theta$ minimisante pour $I_{\alpha}^{*}$.

Nous pouvons résumer ces quelques remarques dans le :

\section{Lemme 2}

(i) $I_{\alpha}^{*}=\inf \left\{E^{*}[\theta] \mid \theta \in X^{*}, \theta(\infty)=0, P(\theta(0))=\frac{\alpha \pi}{2}\right\} \quad(\alpha \in \mathbb{R})$.

(ii) Toute fonction $\theta$ de $X^{*}$ minimisante pour $I_{\alpha}^{*}$ est solution du système : 


$$
\begin{aligned}
& -\frac{d}{d r}\left(r^{2} \dot{\omega}\right)+r^{2} \omega+\sin ^{2} \theta \dot{\theta}=0 \\
& -\frac{d}{d r}\left(r^{2} \dot{\theta}\right)+\sin (2 \theta)+\sin ^{2} \theta \dot{\omega}=0
\end{aligned}
$$

que l'on résoud en $\omega$ par :

$$
\begin{gathered}
\omega(r)=\frac{e^{-r}}{r}\left[P(\theta(0))-P(\theta(r))-\int_{0}^{r} \sin ^{2} \theta(s) \dot{\theta}(s)\left(\frac{s h s}{s}-1\right) d s\right] \\
-\frac{s h r}{r} \int_{r}^{\infty} \sin ^{2} \theta(s) \dot{\theta}(s) \frac{e^{-s}}{s} d s .
\end{gathered}
$$

Enonçons maintenant le résultat principal :

Théorème Pour tout réel $\alpha$, il existe une fonction $\theta \in X^{*} \cap C^{\infty}(] 0, \infty[)$ telle que :

$$
E^{*}[\theta]=I_{\alpha}^{*} \quad \text { et } \quad d^{*}[\theta]=\alpha
$$

Preuve Soit $\alpha \in \mathbb{R}$. Considérons une suite $\left(\theta_{n}\right)_{n \in \mathbb{N}}$, minimisante pour $I_{\alpha}^{*}$ :

$$
\begin{gathered}
d^{*}\left[\theta_{n}\right]=\alpha, \\
\lim _{n \rightarrow \infty} E^{*}\left[\theta_{n}\right]=I_{\alpha}^{*} .
\end{gathered}
$$

Grâce à l'assertion (i) du lemme 2, il n'est pas restrictif de supposer que

$$
\theta_{n}(\infty)=0 \quad \text { et } \quad P\left(\theta_{n}(0)\right)=\frac{\alpha \pi}{2}
$$

Soit $\left(\omega_{n}\right)_{n \in \mathbb{N}}$ la suite associée à $\left(\theta_{n}\right)_{n \in \mathbb{N}}$ par (18) avec $\theta=\theta_{n}$. Par la proposition 2, $\omega_{n}$ est l'unique solution de (10) avec $\theta=\theta_{n}$, telle que : $r \omega_{n} \in L^{2}(] 0, \infty[)$ et $r \dot{\omega}_{n} \in L^{2}(] 0, \infty[)$ et :

$$
\begin{gathered}
\omega(r)=\frac{e^{-r}}{r}\left[\frac{\alpha \pi}{2}-P\left(\theta_{n}(r)\right)-\int_{0}^{r} \sin ^{2} \theta_{n}(s) \dot{\theta_{n}}(s)\left(\frac{s h s}{s}-1\right) d s\right] \\
-\frac{s h r}{r} \int_{r}^{\infty} \sin ^{2} \theta_{n}(s) \dot{\theta_{n}}(s) \frac{e^{-s}}{s} d s .
\end{gathered}
$$

De plus, $\left(\theta_{n}\right)_{n \in \mathbb{N}}$ étant une suite minimisante :

$$
\limsup _{n \rightarrow \infty} \int_{0}^{\infty} r^{2}\left(\omega_{n}^{2}+\dot{\omega}_{n}^{2}\right) d r<I_{\alpha}^{*}
$$

Des arguments de minimisation classiques montrent qu'il existe deux fonctions $\theta$ et $\omega$ de $H_{l o c}^{1}(] 0, \infty[)$ telles que :

$$
\begin{aligned}
& r \dot{\theta}_{n} \rightarrow r \dot{\theta} \\
& r \dot{\omega}_{n} \rightarrow r \dot{\omega} \\
& r \omega \rightarrow r \omega
\end{aligned}
$$

au sens de la topologie $L^{2}(] 0, \infty[)$-faible.

Quitte à extraire des sous-suites, on peut supposer que : 


$$
\begin{aligned}
& \theta_{n} \rightarrow \theta \\
& \omega_{n} \rightarrow \omega
\end{aligned}
$$

au sens presque partout sur $] 0, \infty[$, et même pour tout $r \in] 0, \infty[$, en choisissant les représentants continus (voir remarque 2). D'où en particulier :

$$
\sin \theta_{n} \rightarrow \sin \theta
$$

au sens de la topologie $L_{\text {loc }}^{2}(] 0, \infty[)$-forte, ce qui assure :

$$
\begin{aligned}
\omega(r)=\frac{e^{-r}}{r}[ & \left.\frac{\alpha \pi}{2}-P(\theta(r))-\int_{0}^{r} \sin ^{2} \theta(s) \dot{\theta}(s)\left(\frac{s h s}{s}-1\right) d s\right] \\
& -\frac{s h r}{r} \int_{r}^{\infty} \sin ^{2} \theta(s) \dot{\theta}(s) \frac{e^{-s}}{s} d s .
\end{aligned}
$$

Sous cette forme, $\omega$ est solution de (10), et par la proposition 2 :

$$
\theta \in X^{*} \quad \text { et } \quad P(\theta(0))=\frac{\alpha \pi}{2}
$$

L'estimation :

$$
\left|\theta_{n}(r)\right| \leq \frac{1}{\sqrt{r}} \cdot\left[\int_{0}^{\infty} s^{2} \dot{\theta}_{n}^{2}(s) d s\right]^{1 / 2},
$$

obtenue par le lemme 1, passe à la limite :

$$
\theta(\infty)=0
$$

On a donc démontré que $\theta$ est minimisante pour $I_{\alpha}^{*}$ :

$$
\theta \in X^{*}, \quad d^{*}[\theta]=\alpha \quad \text { et } \quad E^{*}[\theta]=I_{\alpha}^{*}
$$

Il reste à montrer que $\theta$ est de classe $C^{\infty}$ sur $] 0, \infty[$. Pour cela, il suffit de remarquer (assertion (ii) du lemme 2) que $\theta$ et $\omega$ sont solutions du système (S), que $\omega$ est de classe $C^{2}$ sur $] 0, \infty[$ (assertion (ii) de la proposition 2), et que $\theta$ est continue sur $] 0, \infty[$ (lemme 1 ).

Par l'équation (27):

$$
\frac{d}{d r}\left(r^{2} \dot{\theta}\right)=\sin (2 \theta)+\sin ^{2} \theta \dot{\omega},
$$

on obtient que $r^{2} \dot{\theta}(r)$ est de classe $C^{1}$, donc que $\theta$ est de classe $C^{2}$ sur $] 0, \infty[$. En reportant dans l'équation (10) :

$$
\frac{d}{d r}\left(r^{2} \dot{\omega}\right)=r^{2} \omega+\sin ^{2} \theta \dot{\theta}
$$

on obtient que $\omega$ est de classe $C^{3}$ sur $] 0, \infty[$. Il suffit alors d'itérer le procédé pour montrer que $\theta$ et $\omega$ sont de classe $C^{\infty}$ sur $] 0, \infty[$.

Remarque 3 Le théorème montre que toute solution minimisante est de classe $C^{\infty}$ sur ]0, $\infty[$. C'est pourquoi il aurait été possible de définir le problème de minimisation non pas dans l'espace fonctionnel $X^{*}$, mais dans le sous-espace de $X^{*}$ suivant : $Y^{*}=X^{*} \cap C^{\infty}(] 0, \infty[)$. 


\section{Le modèle non symétrique}

Nous allons maintenant essayer d'indiquer quelques-unes des difficultés qui apparaissent lorsque l'on ne fait pas l'hypothèse de symétrie (4).

Introduisons l'espace fonctionnel :

$$
X_{0}=\left\{\phi \in L^{\infty}\left(\mathbb{R}^{3}, S^{3}\right) \mid \nabla \phi \in L^{2}\left(\mathbb{R}^{3}\right)\right\}
$$

Sur $X_{0}$, définissons les fonctionnelles $A$ et $B$ par :

$$
\begin{gathered}
A[\phi]=\left(\partial_{\alpha} \phi \wedge \partial_{\beta} \phi\right)_{\alpha, \beta=1,2,3} \\
B[\phi]=\partial_{1} \phi \wedge \partial_{2} \phi \wedge \partial_{3} \phi
\end{gathered}
$$

Pour toute fonction $\phi$ de $X_{0}, A[\phi]$ et $B[\phi]$ sont des distributions définies au sens suivant : soit $F \in D\left(\mathbb{R}^{3}\right)$

$$
\begin{gathered}
\int_{\mathbb{R}^{3}} F \partial_{\alpha} \phi \wedge \partial_{\beta} \phi \cdot e_{i} \wedge e_{j} d x=\int_{\mathbb{R}^{3}} F\left(\partial_{\alpha} \phi^{i} \partial_{\beta} \phi^{j}-\partial_{\alpha} \phi^{j} \partial_{\beta} \phi^{i}\right) d x \\
(i, j=1,2,3,4 ; \alpha, \beta=1,2,3) \\
\int_{\mathbb{R}^{3}}\left(F \cdot e_{i}\right) \cdot\left(\partial_{1} \phi \wedge \partial_{2} \phi \wedge \partial_{3} \phi\right) d x \\
=- \\
-\frac{1}{3} \int_{\mathbb{R}^{3}}\left(\left(\partial_{1} F \cdot e_{i}\right) \cdot \phi \wedge \partial_{2} \phi \wedge \partial_{3} \phi\right. \\
+\left(\partial_{2} F \cdot e_{i}\right) \cdot \partial_{1} \phi \wedge \phi \wedge \partial_{3} \phi \\
\left.+\left(\partial_{3} F \cdot e_{i}\right) \cdot \partial_{1} \phi \wedge \partial_{2} \phi \wedge \phi\right) d x
\end{gathered}
$$

avec $i=1,2,3,4$ et toutes ces quantités sont bien définies car :

$$
\nabla \phi \in L^{2}\left(\mathbb{R}^{3}\right) \quad .
$$

Intéressons-nous en premier lieu à l'hypothèse de symétrie de manière plus précise, et pour cela, revenons sur les calculs de l'introduction. Soit $\theta \in X^{*} \cap C^{1}\left(\left[0, \infty[)\right.\right.$, et $\phi_{\theta}$ la fonction qui lui est associée par (4) :

$$
\forall x \in \mathbb{R}^{3} \quad \phi_{\theta}(x)=\left(\cos \theta, \frac{x}{r} \sin \theta\right)
$$

Pour tout $\varepsilon>0$, définissons $\theta^{\varepsilon}$ par :

$$
\begin{array}{ll}
\theta^{\varepsilon}(r) & =\left(\frac{r}{\varepsilon}\right)\left[\theta(\varepsilon)+(r-\varepsilon)\left(\dot{\theta}(\varepsilon)-\frac{1}{\varepsilon} \theta(\varepsilon)\right)\right] \\
\theta^{\varepsilon}(r) & =\theta(r) \quad \text { si } r \in[0, \varepsilon] \\
& \text { si } r \in[\varepsilon,+\infty]
\end{array}
$$

$\theta^{\varepsilon}$ est de classe $C^{1}$ sur $\left[0, \infty\left[\right.\right.$ et la fonction $\phi^{\varepsilon}=\phi_{\theta} \varepsilon$ qui lui est associée par (4) est de classe $C^{1}$ sur $\mathbb{R}^{3}$. De plus, quand $\varepsilon \rightarrow 0+$ :

$$
\begin{gathered}
\int_{0}^{\infty} r^{2}\left(\dot{\theta}^{\varepsilon}-\dot{\theta}\right)^{2} d r \rightarrow 0 \\
\int_{0}^{\infty}\left(\sin \theta^{\varepsilon}-\sin \theta\right)^{2} d r \rightarrow 0
\end{gathered}
$$

Or : $\nabla \phi^{\varepsilon} \in L^{2}\left(\mathbb{R}^{3}\right)$ et $:\left\|\nabla \phi^{\varepsilon}\right\|_{L^{2}}^{2}=\int_{0}^{\infty}\left(r^{2}\left(\dot{\theta}^{\varepsilon}\right)^{2}+2 \sin ^{2} \theta^{\varepsilon}\right) d r$. 
A l'extraction d'une sous-suite près, $\nabla \phi^{\varepsilon}$ converge pour la topologie $L^{2}\left(\mathbb{R}^{3}\right)$-faible, et comme :

$$
\phi^{\varepsilon} \rightarrow \phi_{\theta} \quad p p
$$

$\nabla \phi_{\theta} \in L^{2}\left(\mathbb{R}^{3}\right)$, donc : $\phi_{\theta} \in X_{0}$ et $B\left[\phi_{\theta}\right]$ est défini au sens des distributions. De plus :

$$
\lim _{\varepsilon \rightarrow 0+}\left\|\nabla \phi^{\varepsilon}\right\|_{L^{2}}^{2}=\left\|\nabla \phi_{\theta}\right\|_{L^{2}}^{2}
$$

donc :

$$
\nabla \phi^{\varepsilon} \rightarrow \nabla \phi_{\theta} \quad \text { au sens de } \quad L^{2}\left(\mathbb{R}^{3}\right)
$$

et la définition de $B$ montre que :

$$
B\left[\phi^{\varepsilon}\right] \rightarrow B\left[\phi_{\theta}\right] \text { au sens des distributions. }
$$

Il est facile de démontrer que :

$$
B\left[\phi^{\varepsilon}\right](x)=-\frac{1}{r^{2}} \sin ^{2} \theta^{\varepsilon} \cdot \dot{\theta}^{\varepsilon} \cdot\left(\cos \theta^{\varepsilon}, \frac{x}{r} \sin \theta^{\varepsilon}\right) .
$$

Définissons $\tilde{B}$ par :

$$
\tilde{B}\left[\phi_{\theta}\right](x)=-\frac{1}{r^{2}} \sin ^{2} \theta \cdot \dot{\theta} \cdot\left(\cos \theta, \frac{x}{r} \sin \theta\right)
$$

Soit $F \in D\left(\mathbb{R}^{3}\right)$ :

$$
\begin{aligned}
\int_{\mathbb{R}^{3}} F \cdot B\left[\phi^{\varepsilon}\right] d x \cdot e^{i}=0 \quad \text { pour } i=2,3,4 \\
\int_{\mathbb{R}^{3}} F \cdot B\left[\phi^{\varepsilon}\right] d x \cdot e^{1} \\
\quad \sim 4 \pi F(0) \cdot \int_{0}^{\varepsilon}\left(\sin ^{2} \theta^{\varepsilon} \cos \theta^{\varepsilon} \dot{\theta}^{\varepsilon}-\sin ^{2} \theta \cos \theta \dot{\theta}\right) d r \\
\rightarrow-4 \pi F(0) \sin ^{3} \theta(0) \quad \text { quand } \varepsilon \rightarrow 0+
\end{aligned}
$$

d'où :

$$
B\left[\phi_{\theta}\right]=\tilde{B}\left[\phi_{\theta}\right]-2 \pi \sin ^{3} \theta(0) \delta \cdot e_{1} .
$$

Remarquons que pour toute fonction $\phi$ de $X_{0}, \phi$ est à valeurs dans $S^{3}$ presque partout, donc :

$$
\phi \cdot \partial_{\alpha} \phi=0 \quad \text { pp. } \quad(\alpha=1,2,3)
$$

Par conséquent, pour que :

$$
\operatorname{det}\left(\phi_{\theta}, \nabla \phi_{\theta}\right)=\phi_{\theta} \cdot B\left[\phi_{\theta}\right]
$$

appartienne à $L^{1}\left(\mathbb{R}^{3}\right)$, il faut et il suffit que ,

$$
\mid \operatorname{det}\left(\phi_{\theta}, \nabla \phi_{\theta}|=| B\left[\phi_{\theta}\right] \mid \in L^{1}\left(\mathbb{R}^{3}\right)\right.
$$

c'est-à-dire :

$$
\theta(0) \in \pi
$$

La proposition A3 de l'appendice montre qu'alors $d^{*}[\theta]$ est le degré au sens usuel de $\phi_{\theta}$. Résumons ces quelques propriétés dans la :

Proposition 3 Soit $\theta \in X^{*} \cap C^{1}\left(\left[0, \infty[)\right.\right.$, et $\phi_{\theta}$ la fonction qui lui est associée par (4). Alors :

(i) $\phi_{\theta} \in X_{0}$. 
(ii)

$$
B\left[\phi_{\theta}\right]=\tilde{B}\left[\phi_{\theta}\right]-4 \pi \sin ^{3} \theta(0) \delta \cdot e_{1}(35) \text { avec : }
$$

$$
\tilde{B}\left[\phi_{\theta}\right](x)=-\frac{1}{r^{2}} \sin ^{2} \theta \dot{\theta}\left(\cos \theta, \frac{x}{r} \sin \theta\right)
$$

(iii) $\quad\left(\operatorname{det}\left(\phi_{\theta}, \nabla \phi_{\theta}\right) \in L^{1}\left(\mathbb{R}^{3}\right)\right) \Longleftrightarrow(\theta(0) \in \pi)$.

Intéressons-nous maintenant à la formulation du problème sans hypothèse de symétrie et remarquons que l'on ne peut pas définir $E[\phi]$ et $d[\phi]$ pour toute fonction $\phi$ de $X_{0}$. Car si la propriété : $\phi \in X_{0}$ suffit à définir $B[\phi]$ au sens des distributions, elle n'assure pas a priori - comme nous venons de le voir - que : $\operatorname{det}(\phi, \nabla \phi) \in L^{1}\left(\mathbb{R}^{3}\right)$, ce qui est nécessaire pour définir $d[\phi]$, ni même : $\operatorname{det}(\phi, \nabla \phi) \in D^{\prime}\left(\mathbb{R}^{3}\right)$, ce qui est nécessaire pour que l'équation (2) ait un sens : considérons en effet l'exemple suivant :

Soit $\theta \in C^{0}(] 0, \infty[)$ et $\left(\theta_{n}\right)_{n \in \mathbb{N}}$ une suite de fonctions de $X^{*}$, définies par :

$$
\begin{aligned}
\theta(t) & =l n t \quad \text { si } t \in] 0,1[ \\
\theta(t) & =0 \quad \text { si } t \in[1, \infty[ \\
\text { et }: & \\
\theta_{n}(t) & =-n \pi \quad \text { si } t \in\left[0, e^{-n \pi}\right] \\
\theta_{n}(t) & =l n t \quad \text { si } t \in\left[e^{-n \pi}, 1\right] \\
\theta_{n}(t) & =0 \quad \text { si } t \in[1, \infty[
\end{aligned}
$$

On note : $\phi_{\theta}$ et $\phi^{n}=\phi_{\theta^{n}}$ les fonctions associées par (4) à $\theta$ et $\theta_{n}$ respectivement. $\nabla \phi^{n} \in X_{0}$, et : $\nabla \phi^{n} \rightarrow \nabla \phi_{\theta}$ pour la topologie $L^{2}\left(\mathbb{R}^{3}\right): \phi_{\theta} \in X_{0}$. Pourtant, étant donnée $f \in D\left(\mathbb{R}^{3}\right)$, on montre que :

$$
\int_{\mathbb{R}^{3}} f(x) \operatorname{det}\left(\phi^{n}, \nabla \phi^{n}\right) d t \sim-\frac{1}{2} n \pi f(0) \quad(\text { quand } n \rightarrow \infty)
$$

ce qui ne permet pas de définir $\operatorname{det}\left(\phi_{\theta}, \nabla \phi_{\theta}\right)$ au sens des distributions.

Pour définir le problème de minimisation associé au modèle d'Adkins et Nappi sans hypothèse de symétrie, on est donc amené à considérer un sous-espace de $X_{0}$, par exemple :

$$
\begin{gathered}
\bar{X}=\left\{\phi \in X_{0} \mid B[\phi] \in L^{2}\left(\mathbb{R}^{3}\right)\right\} \\
\bar{X}=\left\{\phi \in L^{\infty}\left(\mathbb{R}^{3}, S^{3}\right) \mid \nabla \phi \in L^{2}\left(\mathbb{R}^{3}\right) \text { et } B[\phi] \in L^{2}\left(\mathbb{R}^{3}\right)\right\} .
\end{gathered}
$$

L'équation (2) :

$$
-\Delta \Omega+\Omega=\operatorname{det}(\phi, \nabla \phi)
$$

possède une solution unique dans $H^{1}\left(\mathbb{R}^{3}\right)$ pour tout $\phi \in \bar{X}$, car :

$$
|\operatorname{det}(\phi, \nabla \phi)|=|\phi \cdot B[\phi]| \leq|B[\phi]| \in L^{2}\left(\mathbb{R}^{3}\right) .
$$

La fonctionnelle $E$ est donc bien définie sur $\bar{X}$.

En utilisant les inégalités de Hölder, on montre que :

$$
\begin{aligned}
\int_{\mathbb{R}^{3}}|\operatorname{det}(\phi, \nabla \phi)| d x & =\int_{\mathbb{R}^{3}}|B[\phi]| d x \\
& \leq\|\nabla \phi\|_{L^{2}}^{3 / 2} \cdot\|B[\phi]\|_{L^{2}}^{3 / 2} .
\end{aligned}
$$

La fonctionnelle $d$ est donc bien définie sur $\bar{X}$. 
Nous allons voir que l' espace fonctionnel $\bar{X}$ n' est pourtant pas un espace approprié pour définir le problème de minimisation.

Commençons par étudier l' effet des dilatations : considérons la fonction $\phi^{\lambda} \in \bar{X}$, définie par :

$$
\phi^{\lambda}(x)=\phi\left(\frac{x}{\lambda}\right) \quad \forall x \in \mathbb{R}^{3}
$$

Soit $\Omega^{\lambda}$ la fonction associée à $\phi^{\lambda}$ par :

$$
-\Delta \Omega^{\lambda}+\Omega^{\lambda}=\operatorname{det}\left(\phi^{\lambda}, \nabla \phi^{\lambda}\right)
$$

On peut montrer que quand $\lambda \rightarrow+\infty$ :

$$
\left\|\Omega^{\lambda}\right\|_{H^{1}}^{2} \sim \frac{1}{\lambda^{3}}\|B[\phi]\|_{L^{2}}^{2}=\left\|B\left[\phi^{\lambda}\right]\right\|_{L^{2}}^{2}
$$

Ceci ne suffit pourtant pas à contrôler le terme: $\|B[\phi]\|_{L^{2}}$.

Etudions maintenant de plus près la fonction $\Omega$ lorsque $\phi \in \bar{X}$. Par transformation de Fourier, on obtient pour $\Omega$ l' expression suivante :

$$
\Omega(x)=\frac{1}{(2 \pi)^{3}} \cdot \int_{\mathbb{R}^{3}} d y \frac{e^{-i x y}}{|y|^{2}+1} \int_{\mathbb{R}^{3}} d z e^{i z y} \operatorname{det}(\phi, \nabla \phi)(z)
$$

ou encore :

$$
\Omega(x)=\frac{1}{4 \pi} \cdot \int_{\mathbb{R}^{3}} \frac{e^{-|x-z|}}{-|x-z|} \operatorname{det}(\phi, \nabla \phi)(z) d z \quad .
$$

Il est alors facile de voir que: $\Delta \Omega \in L^{2}\left(\mathbb{R}^{3}\right)$ (d'où en particulier $\Omega \in L^{\infty}\left(\mathbb{R}^{3}\right)$ ) et que :

$$
\|\Delta \Omega\|_{L^{2}}^{2}+2\|\nabla \Omega\|_{L^{2}}^{2}+\|\Omega\|_{L^{2}}^{2}=\|B[\phi]\|_{L^{2}}^{2}
$$

Finalement, au vu de (44) et (47), il est facile de montrer que pour poser le problème de minimisation dans $\bar{X}$, il faut pouvoir contrôler le terme : $\|B[\phi]\|_{L^{2}}$, ce qui revient à modifier la fonctionnelle énergie en lui ajoutant un terme du type :

$$
\|\Delta \Omega\|_{L^{2}}^{2}
$$

ou du type :

$$
\|B[\phi]\|_{L^{2}}^{2}
$$

Ceci modifie complètement la structure du problème. Par ailleurs, il serait naturel de demander que la fonctionnelle degré soit à valeurs entières uniquement, ce qui n'est pas assuré à priori (on ne connaît pas de résultat de densité de fonctions régulières dans $\bar{X}$ : voir [E4]).

$\bar{X}$ n'est donc pas un espace fonctionnel adapté au problème, et le choix d'un espace fonctionnel approprié $X$ reste donc un problème ouvert.

\section{Appendice : degré et champs de mésons}


Nous allons pour finir donner quelques résultats simples relatifs au degré pour l'espace fonctionnel suivant $\left(X_{0}\right.$ est le plus grand espace fonctionnel sur lequel on peut définir les champs de pions utilisés en physique de la matière hadronique) :

$$
X_{0}=\left\{\phi \in L^{\infty}\left(\mathbb{R}^{3}, S^{3}\right) \mid \nabla \phi \in L^{2}\left(\mathbb{R}^{3}\right)\right\} .
$$

Voici tout d'abord un résultat concernant le comportement à l'infini :

Proposition A1 Il existe une constante $C$, positive, telle que, pour toute fonction $\phi$ de $X$, il existe un point unique $P$ de $S^{3}$ pour lequel on a :

$$
\|\phi-P\|_{L^{6}\left(\mathbb{R}^{3}\right)} \leq C \cdot\|\nabla \phi\|_{L^{2}\left(\mathbb{R}^{3}\right)}
$$

En particulier : $(\phi-P) \in L^{6}\left(\mathbb{R}^{3}\right)$.

Preuve Par l'inégalité de Poincaré-Wirtinger appliquée à la boule unité $B$ de $\mathbb{R}^{3}$, il existe une constante $C$ positive telle que l'on ait:

$$
\forall \psi \in W^{1,2}(B) \quad\|\psi-\bar{\psi}\|_{L^{6}(B)} \leq C \cdot\|\nabla \psi\|_{L^{2}(B)}
$$

où :

$$
\bar{\psi}=\frac{1}{|B|} \cdot \int_{B} \psi(s) d x
$$

Soit $\phi \in X$. Pour tout réel $R>0$, on applique $(\mathrm{A} 3)$ à $\psi(\cdot)=\phi(R \cdot)$

$$
\|\phi-P(R)\|_{L^{6}(B(R))} \leq C\|\nabla \phi\|_{L^{2}(B(R))}
$$

où :

$$
P(R)=\bar{\psi}=\frac{1}{|B(R)|} \int_{B(R)} \phi(x) d x .
$$

$|P(R)| \leq\|\phi\|_{L^{\infty}\left(\mathbb{R}^{3}\right)}=1$, et par conséquent, la suite $(P(R))_{R>0}$ possède un point d'accumulation, que l'on notera $P$. Du lemme de Fatou, on déduit que :

$$
\|\phi-P\|_{L^{6}\left(\mathbb{R}^{3}\right)} \leq C \cdot\|\nabla \phi\|_{L^{2}\left(\mathbb{R}^{3}\right)} .
$$

L'unicité de $P$ est évidente et $P \in S^{3}$, sinon on aurait :

$$
\forall x \in \mathbb{R}^{3} \quad|\phi(x)-P|>1-|P|>0 \quad,
$$

ce qui serait contradictoire avec la propriété :

$$
(\phi-P) \in L^{6}\left(\mathbb{R}^{3}\right)
$$

La proposition A1 montre que toute fonction de $X_{0}$ est constante - dans un sens faible - au voisinage de l'infini. Ceci suggère d'étendre la notion de degré à l'aide, par exemple, d'une formule intégrale : nous montrons ci-dessous que l'expression de $d$ possède la forme requise.

Proposition A2 Soit $\phi$ une fonction de $X_{0}$, de classe $C^{1}$, constante en dehors d'un compact, telle que : $\operatorname{det}(\phi, \nabla \phi) \in L^{1}\left(\mathbb{R}^{3}\right)$. Alors :

$$
d[\phi]=\frac{1}{2 \pi^{2}} \int_{\mathbb{R}^{3}} \operatorname{det}(\phi, \nabla \phi) d x
$$

est égal au degré de $\phi$. En particulier, $d[\phi]$ est entier. 
Preuve Soit $\phi$ une fonction vérifiant les hypothèses de la proposition A2 et $P$ le point $S^{3}$ qui lui est associé par la proposition A1. Considérons les fonctions :

$$
\begin{gathered}
\psi: \overline{B^{4}} \rightarrow \mathbb{R}^{4} \\
\tilde{\phi}: \mathbb{R}^{3} \times[0,1] \rightarrow \mathbb{R}^{3}
\end{gathered}
$$

(où $B^{4}$ désigne la boule unité ouverte de $\mathbb{R}^{4}$ ) définies par :

$$
\begin{aligned}
& \psi\left(y^{1}, y^{2}, y^{3}, y^{4}\right)=\tilde{\phi}\left(x^{1}, x^{2}, x^{3}, x^{4}\right) \quad \text { si } y^{4} \neq|y| \\
& \psi\left(0,0,0, y^{4}\right)=P \quad \text { si } y^{4} \geq 0 \\
& \tilde{\phi}\left(x^{1}, x^{2}, x^{3}, x^{4}\right)=\left(x^{4}\right)^{2} \phi\left(x^{1}, x^{2}, x^{3}\right)
\end{aligned}
$$

où le changement de variable $x(y)$ est défini par :

$$
\begin{aligned}
& x^{i}=\frac{2 y^{i}}{|y|-y^{4}} \quad(i=1,2,3) \quad \text { pour tout } y=\left(y^{1}, y^{2}, y^{3}, y^{4}\right) \in \overline{B^{4}} \quad \text { tel que } y^{4} \neq|y| \\
& x^{4}=|y|
\end{aligned}
$$

$\psi$ est de classe $C^{1}$ sur $B^{4}$, continue sur $\overline{B^{4}}$, à valeurs dans $\overline{B^{4}}$ et :

$$
\psi\left(\overline{B^{4}}\right)-\psi\left(S^{3}=\partial \overline{B^{4}}\right)=\psi\left(B^{4}\right)
$$

est étoilé par rapport à l'origine de $\mathbb{R}^{4}$, donc connexe. Le degré de $\psi$ est donc constant sur $\psi\left(B^{4}\right)$ et vaut :

$$
\begin{aligned}
\frac{1}{\left|B^{4}\right|} \int_{B^{4}} \operatorname{det}\left(\frac{\partial \psi}{\partial y^{1}}, \frac{\partial \psi}{\partial y^{2}}, \frac{\partial \psi}{\partial y^{3}}, \frac{\partial \psi}{\partial y^{4}}\right) d y \\
=\frac{1}{\left|B^{4}\right|} \int_{\mathbb{R}^{3} \times[0,1]} \operatorname{det}\left(\frac{\partial \tilde{\phi}}{\partial x^{1}}, \frac{\partial \tilde{\phi}}{\partial x^{2}}, \frac{\partial \tilde{\phi}}{\partial x^{3}}, \frac{\partial \tilde{\phi}}{\partial x^{4}}\right) d x^{1} d x^{2} d x^{3} \cdot d x^{4} \\
=\frac{2}{\pi^{2}} \int_{\mathbb{R}^{3} \times[0,1]} 2\left(x^{4}\right)^{7} \operatorname{det}\left(\phi, \frac{\partial \phi}{\partial x^{1}}, \frac{\partial \phi}{\partial x^{2}}, \frac{\partial \phi}{\partial x^{3}}\right) d x \cdot d x^{4} \\
=12 \pi^{2} \int_{\mathbb{R}^{3}} \operatorname{det}(\phi, \nabla \phi) d x
\end{aligned}
$$

Dans le cadre de l'ansatz de Skyrme, nous pouvons énoncer un résultat plus précis encore. Considérons une fonction de la forme :

$$
\forall x \in \mathbb{R}^{3} \quad \phi_{\theta}(x)=(\cos \theta, x r \sin \theta)
$$

où $\theta$ vérifie les conditions usuelles, à savoir,

$$
\theta \in H_{l o c}^{1}(] 0, \infty[) \cap C^{0}\left(\left[0, \infty[) \quad, \quad \sin \theta \in L^{2}(] 0, \infty[) \quad .\right.\right.
$$

Lorsque : $\theta(0) \in \pi, \phi_{\theta}$ est continue sur $\mathbb{R}^{3}$, et comme $\phi_{\theta}$ possède une limite au voisinage de l'infini (par le lemme 1), le degré est défini au sens usuel et il est égal à : $d^{*}[\theta]$.

Proposition A3 Soit $\theta \in H_{l o c}^{1}(] 0, \infty[) \cap C^{0}([0, \infty[)$, telle que $\theta(0) \in \pi$ et :

$$
\begin{gathered}
r \dot{\theta} \in L^{2}(] 0, \infty[) \\
\sin \theta \in L^{2}(] 0, \infty[)
\end{gathered}
$$

Alors $d^{*}[\theta]$ est le degré, au sens usuel, de la fonction $\phi_{\theta}$ définie par (4). 
Preuve Par troncature, on se ramène au cas où $\theta$ est à support compact (sans changer le degré de $\phi_{\theta}$, grâce à la proposition A1); de même, on peut supposer que $\theta$ est constante dans un voisinage de $r=0+$. Soit $\varphi$ la fonction définie sur $\mathbb{R}^{3}$ par :

$$
\forall x \in \mathbb{R}^{3} \quad \varphi(x)=\theta(r) \quad \text { avec } \quad r=|x|
$$

et $\left(\varphi^{\varepsilon}\right)_{\varepsilon>0}$ une suite de fonctions régularisante pour $\varphi$, obtenue par convolution avec une fonction radiale de classe $C^{1}$. Il est facile de voir que pour tout $\varepsilon>0, \varphi^{\varepsilon}$ est radiale : définissons sur $\left[0, \infty\left[\right.\right.$ la fonction $\theta^{\varepsilon}$ par:

$$
\forall x \in \mathbb{R}^{3} \quad \theta^{\varepsilon}(r)=\varphi^{\varepsilon}(x)
$$

$\phi_{\theta^{\varepsilon}}$ converge vers $\phi_{\theta}$ uniformément, et $\phi_{\theta^{\varepsilon}}$ est de classe $C^{1}$ (car $\theta^{\varepsilon}$ est constante dans un voisinage de $r=0+$ ) pour $\varepsilon$ assez petit. Par la proposition A2, le degré de $\phi_{\theta^{\varepsilon}}$ est égal à $d\left[\phi_{\theta^{\varepsilon}}\right]=d^{*}\left[\theta^{\varepsilon}\right]$ et $d^{*}\left[\theta^{\varepsilon}\right]=d^{*}[\theta]$, ce qui démontre la proposition.

\section{Bibliographie}

[S] T.H.R. Skyrme, A non-linear field theory. Proc. Roy. Soc., A 260 (1961) 127-138.

$[\mathrm{A}, \mathrm{N}, \mathrm{W}]$ G.S. Adkins, C.R. Nappi, E. Witten, Static properties of the nucleons in the Skyrme model. Nucl. Phys., B 228 (1983) 552-596.

[A,N] G.S. Adkins, C.R. Nappi, Stabilization of chiral solitons via vector mesons. Phys. Lett., vol. 67 B, numb. 3,4 (1984) 251-256.

[M,K,W,W] U.G. Meissner, N. Kaiser, A. Wirzba, W. Weise,Skyrmions with $\rho$ et $\omega$ mesons as dynamical gauge bosons. Phys. Rev. Lett., vol. 57, numb. 14 (1986), 1676-1679.

[M,Z] U.G. Meissner, I. Zahed, Nucleons from Skyrmions with vector mesons. Z. Phys. A. Atomic Nuclei 327 (1987) 5-15.

[VM] R. Vinh Mau, Skryme solitons and effective Lagrangians for low energy hadronic physics. Preprint IPNO/TH 87178 (1987).

[E1] M.J. Esteban, An isoperimetric inequality in $\mathbb{R}^{3}$. Ann. Inst. Henri Poincaré, vol. 4, numb. 4 (1987) 297-305.

[E2] M.J. Esteban, Existence of symmetric solutions for the Skyrme's problem. Annali di matematica pura ed applicata (iv), vol. CXLVII (1987) 187-195.

[E3] M.J. Esteban, A direct variational approach to Skyrme's model for meson fields. Comm. Math. Phys. 105 (1987) 571-591.

[E4] M.J. Esteban, A new setting for the Skyrme's problem.à paraître dans : Proc. Coll. "Problèmes variationnels" (Paris, juin 1988) ed. Beresticky, Coron, Ekeland. Birkhäuser.

[L] P.L. Lions, The concentration-compactness principle in the calculus of variations. The limit case. Part I : Rev. Mat. Iber. I,1 (1985) 145-200. Part II : Rev. Math. Iber. I,2 (1985) 45-121. 